OPEN ACCESS

Edited by:

Xian Wu,

Miami University, United States

Reviewed by:

Xiaogiong Cao,

University of Massachusetts Amherst

United States

Jianan Zhang,

University of Massachusetts Amherst,

United States

${ }^{*}$ Correspondence:

Azita Hekmatdoost

a_hekmat2000@yahoo.com

Specialty section:

This article was submitted to

Nutritional Immunology,

a section of the journal

Frontiers in Nutrition

Received: 14 August 2021 Accepted: 18 November 2021

Published: 16 December 2021

Citation:

Rastgoo S, Ebrahimi-Daryani N

Agah S, Karimi S, Taher M

Rashidkhani B, Hejazi E, Mohseni F,

Ahmadzadeh M, Sadeghi A and

Hekmatdoost A (2021) Glutamine

Supplementation Enhances the

Effects of a Low FODMAP Diet in

Irritable Bowel Syndrome

Management. Front. Nutr. 8:746703.

doi: 10.3389/fnut.2021.746703

\section{Glutamine Supplementation Enhances the Effects of a Low FODMAP Diet in Irritable Bowel Syndrome Management}

\author{
Samira Rastgoo ${ }^{1}$, Nasser Ebrahimi-Daryani ${ }^{2}$, Shahram Agah ${ }^{3}$, Sara Karimi ${ }^{2}$, \\ Mohammad Taher ${ }^{2}$, Bahram Rashidkhani ${ }^{1}$, Ehsan Hejazi ${ }^{1}$, Fatemeh Mohseni ${ }^{1}$, \\ Mina Ahmadzadeh ${ }^{1}$, Amir Sadeghi ${ }^{4}$ and Azita Hekmatdoost ${ }^{1 *}$
}

${ }^{1}$ Department of Clinical Nutrition and Dietetics, Faculty of Nutrition and Food Technology, National Nutrition and Food Technology, Research Institute, Shahid Beheshti University of Medical Sciences, Tehran, Iran, ${ }^{2}$ Department of Gastroenterology and Hepatology, Tehran University of Medical Sciences, Tehran, Iran, ${ }^{3}$ Colorectal Research Center, Iran University of Medical Sciences, Tehran, Iran, ${ }^{4}$ Gastroenterology and Liver Diseases Research Center, Research Institute for Gastroenterology and Liver Diseases, Shahid Beheshti University of Medical Sciences, Tehran, Iran

Background and Aims: Although irritable bowel syndrome is one of the most common gastrointestinal disorders presented to gastroenterologists, therapeutic strategies are not yet well-established. Accordingly, we conducted a randomized, double-blind, placebo-controlled, clinical trial to evaluate the possible superiority of adding glutamine supplement to low fermentable oligo- di- monosaccharides and polyols (FODMAP) diet in patients with irritable bowel syndrome (IBS).

Methods: Eligible adults were randomized to receive a low FODMAP diet either with glutamine (15 g/day) or a placebo for 6 weeks. The primary endpoint was a significant reduction in IBS-symptom severity score (IBS-SSS). Secondary endpoints were changes in IBS symptoms, stool frequency, consistency, and quality of life.

Results: The study group enrolled 50 patients, among which 22 participants from each group completed the study protocol. The glutamine group had significant changes in total IBS-severity score, dissatisfaction of bowel habit and interference with community function (58\% reduction; $P<0.001,57 \%$ reduction; $P<0.001,51 \%$ reduction; $P=0.043$, respectively). Improvement in IBS-severity score of more than $45 \%$ was observed in 22 of 25 participants (88\%) in the glutamine group, while it was only 15 of 25 participants $(60 \%)$ in the control group $(p=0.015)$. No serious adverse events were observed.

Conclusions: Our findings indicated the superiority of adding glutamine supplementation to a low FODMAP diet in amelioration of IBS symptoms while confirming the beneficial effects of a low FODMAP diet in IBS management.

Keywords: irritable bowel syndrome (IBS), glutamine, diet, low FODMAP diet, clinical trial 


\section{INTRODUCTION}

Although irritable bowel syndrome is one of the most prevalent referrals to gastroenterologists, the best method for managing it is still unknown $(1,2)$. The high prevalence of irritable bowel syndrome (IBS) besides suboptimal medical treatments leads to significant economic costs and psychosocial burden (3-5). The pathophysiology of IBS is multifactorial and the molecular mechanisms underlying the pathophysiology of IBS are not well-understood. However, dietary intolerance, alternation in gut microbiota, and increased intestinal permeability have been suggested as potential risk factors $(3,6,7)$. Recent studies have shown that a diet low in fermentable oligosaccharides, disaccharides, monosaccharides, and polyols (FODMAPs) can improve gastrointestinal symptoms in patients with IBS (8-10); however, the patients on low FODMAPs diet are not completely free of symptoms (11). Moreover, the results of previous studies have mainly reported the efficacy of a low FODMAP diet on pain and bloating reduction, with no effect on stool consistency and frequency (8). Thus, it seems that we still need new strategies for IBS management with a special focus on stool consistency and frequency.

Glutamine is a non-essential amino acid that is a preferred energy source for cells with rapid turnover such as lymphocytes and enterocytes. This amino acid promotes enterocyte proliferation, regulates tight junction proteins, and suppresses pro-inflammatory signaling pathways (12). It has been reported that the increased intestinal permeability that occurs in diarrheapredominant patients with IBS might be due to decreased glutamine synthetase levels (13). Meanwhile; experimental evidence suggests that glutamine supplementation reduces intestinal permeability $(14,15)$. Furthermore, glutamine supplementation changes the intestinal microenvironment and regulates intestinal bacteria's utilization and metabolism of amino acids, thereby altering the composition of intestinal microbiota $(16,17)$. Modulation of intestinal microbiota might ameliorate constipation and improve intestinal function $(18,19)$.

In light of this experimental evidence, we hypothesized that the co-administration of a low FODMAP diet and an oral glutamine supplement would reduce the symptoms and improve the quality of life of patients with IBS more effectively than a low FODMAP diet alone. Thus, the current randomized, doubleblind, placebo-controlled trial was conducted to evaluate the effect of a low FODMAP diet with glutamine supplementation on patients with IBS' clinical outcomes and quality of life.

\section{METHODS AND MATERIALS}

\section{Participants}

This study's participants comprised patients with IBS (as defined by Rome IV criteria) (20) without any other disorders aged between 18 and 70 years old with body mass index (BMI) ranging from 18.5 to 25 . Patients were recruited between June 2020 and December 2020 from two gastroenterology clinics in Tehran, Iran. Patients who did not have the following disorders were eligible to participate in the study: any organic intestinal diseases based on colonoscopy over the past 5 years, intestinal infection, history of colorectal disorders, major intestinal surgery, liver, kidney, psychiatric disease, or any GI disease other than IBS.

Patients were excluded from the study if they (a) were taking any medication with antispasmodics, antibiotics, anti-diarrhea or laxative properties, prokinetics, non-steroidal anti-inflammatory, and immunosuppressive agents or (b) were a smoker, pregnant, or breastfeeding. Patients with known allergies to glutamine or whey protein were excluded, as were those who were taking or had taken supplements containing glutamine or whey protein. Patients were also excluded if they had consumed synthetic sweeteners within 2 days before the study or during the study, as such sweeteners can alter intestinal permeability. Finally, any patients unwilling to adhere to the recommended diet were also excluded.

Patients were sub-classified as either having predominant diarrhea (IBS-D), predominant constipation (IBS-C), mixed or alternating bowel habits (IBS-M), or undetermined categories (IBS-U). All patients provided informed written consent to participate in the study after the study protocol was thoroughly explained.

\section{Study Design and Intervention}

This study was a randomized, double-blind, placebo-controlled trial. Fifty patients who met the inclusion criteria were randomly assigned to either the experimental (glutamine) or control (placebo) group. Randomization was based on a random table sequence, and all investigators were blind regarding which patients were in which group.

Since receiving $15 \mathrm{~g}$ of glutamine or whey does not cause side effects for patients (21), participants received an oral glutamine powder or placebo powder (whey protein) at a dose of $15 \mathrm{~g} \mathrm{(5 \textrm {g }}$ mixed in water three times per day) for 6 weeks. The powders were similar in color, consistency, and taste. The supplements were concealed as A or B by a third party-for the duration of the study, neither the participants nor investigators knew which group was taking which supplement.

All participants were advised to follow a low FODMAP diet in addition to taking the assigned supplement. Diets were administered by an experienced dietitian according to the guidelines of the National Institute for Health and Care Excellence (NICE) while omitting high FODMAPs foods. All diets contained $<5$-g FODMAPs per day. Patients' adherence to the diet was evaluated by recording of 3 days ( 1 weekend and 2 workdays) dietary recalls at week 2 and the end of the study.

Dietary intakes of FODMAPs were assessed using Monash University's low-FODMAP diet database and quantitative reports of FODMAP content in recent studies $(22,23)$. Patients were followed by phone calls to assess protocol adherence, record any supplement side effects, and answer any study-related questions. Patients' competency was assessed using the measurement of remained supplements.

\section{Study Outcomes}

The primary outcome was a significant reduction in IBSsymptom severity score (IBS-SSS). Secondary outcomes were changes in IBS symptoms, quality of life, and stool consistency and frequency. All clinical outcomes were evaluated at baseline 


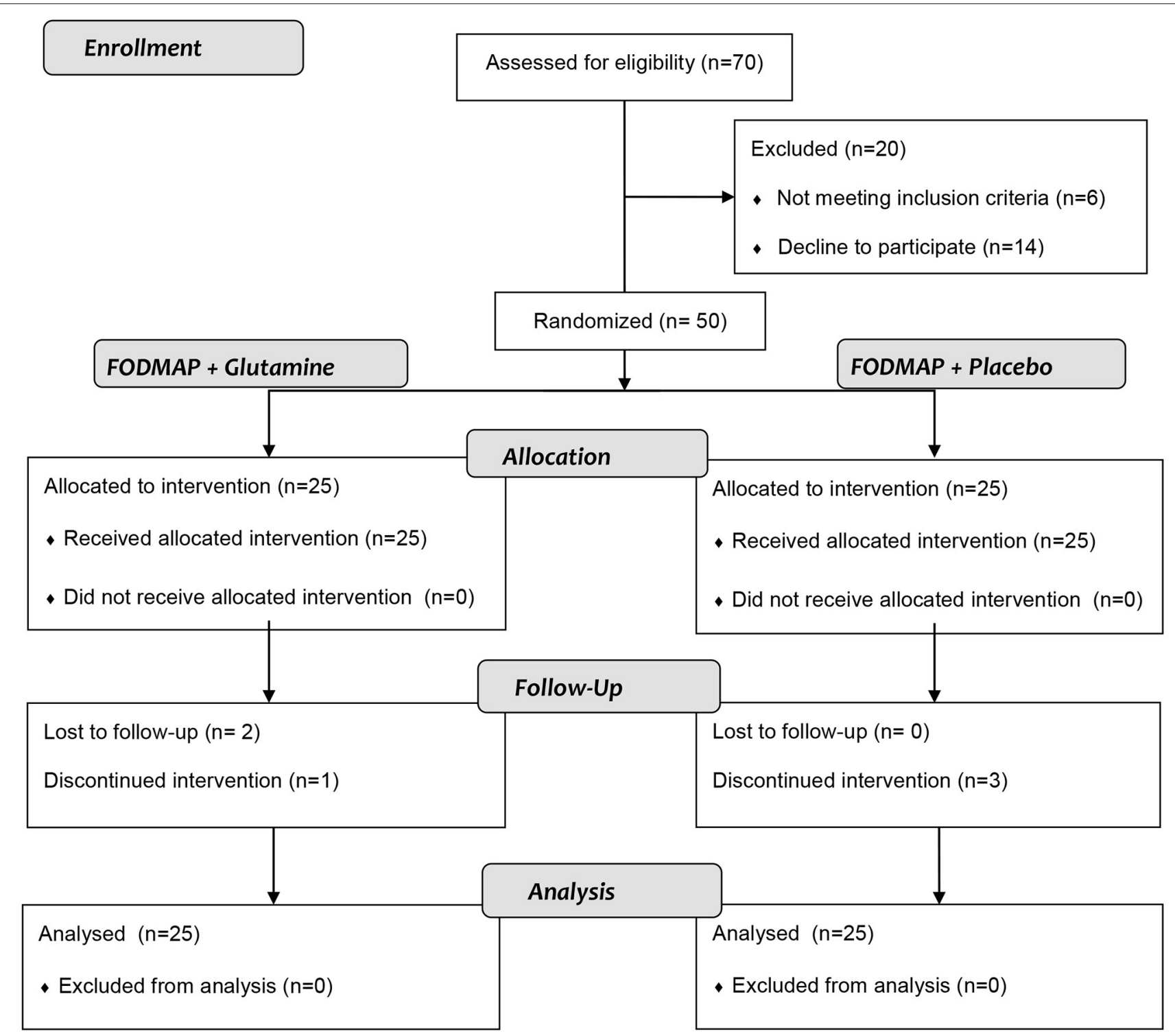

FIGURE 1 | Consort flow chart of the study.

and the end of the study using the IBS-SSSQ (24). This instrument includes five clinically relevant items that determine the severity of abdominal pain, the frequency of pain, abdominal distension, satisfaction with bowel habits, and the interference of IBS with community function as measured on the visual analog scale (VAS) (with an array of $100 \mathrm{~mm}$, with 0 indicating no symptoms and 100 representing extremely severe symptom). The sum of these five items was IBS- symptom severity score (range $=0-500)$. Scores of 75-175, 175-300, and $>300$ indicated mild, moderate, and severe cases, respectively.

Each patient's quality of life (QoL) was assessed at baseline and the end of the study using a 34-item self-report measure specific to IBS (IBS-QoL) (25). Each item was answered on a 5-point Likert scale, and participants' scores were summed to derive the overall score. Scores were subsequently transposed onto a scale from 0 to 100, with higher scores indicating a better quality of life.

Stool consistency was assessed using the validated Bristol Stool Form Scale (BSFS) (26). Stool frequency (i.e., number of stools per day) was recorded at baseline and the end of the study.

\section{Statistical Analysis}

The sample size was calculated based on the formula by considering $\alpha$ (type 1 error) $=0.05$ and power of $80 \%$ according to the published article (11), which was obtained 21. Therefore, according to the formula 21 subjects were needed in each group, considering the probable dropouts, we assigned 25 subjects for each group to meet the adequate power. 
TABLE 1 | Characteristics of participants with irritable bowel syndrome at the baseline.

\begin{tabular}{|c|c|c|c|}
\hline Baseline characteristics & Glutamine $(n=25)$ & Control $(n=25)$ & $P$-value \\
\hline Age (years) $^{a}$ & $40.36 \pm 15.15$ & $35.09 \pm 8.53$ & 0.256 \\
\hline Sex-no. $(\%)^{b}$ & & & 0.540 \\
\hline Female & 14 (56\%) & 16 (64\%) & \\
\hline Male & 11 (44\%) & $9(36 \%)$ & \\
\hline Weight $(\mathrm{kg})^{\mathrm{a}}$ & $67.36 \pm 8.44$ & $63.09 \pm 9.61$ & 0.125 \\
\hline Constipate & $3(12 \%)$ & $4(16 \%)$ & \\
\hline Diarrhea & $16(64 \%)$ & $14(56 \%)$ & \\
\hline mixed & $6(24 \%)$ & $4(16 \%)$ & \\
\hline Unclassified & 0 & $3(12 \%)$ & \\
\hline \multicolumn{4}{|l|}{ IBS symptoms ${ }^{a}$} \\
\hline Abdominal distension & $56.14 \pm 22.30$ & $66.82 \pm 12.20$ & $0.292^{*}$ \\
\hline dissatisfaction with bowel habits & $64.32 \pm 29.93$ & $49.77 \pm 20.73$ & 0.068 \\
\hline Interference with life & $69.32 \pm 22.43$ & $70.91 \pm 10.19$ & $0.971^{*}$ \\
\hline Stool frequency (no./day) & $2.95 \pm 2.19$ & $2.68 \pm 2.05$ & $0.685^{\star}$ \\
\hline Stool consistency & $5.14 \pm 1.70$ & $4.55 \pm 1.62$ & $0.240^{*}$ \\
\hline Quality of life ${ }^{a}$ & $63 \pm 19.99$ & $65 \pm 10.44$ & 0.652 \\
\hline
\end{tabular}

${ }^{a}$ Data are reported as mean $\pm S D$ and compared by independent sample $t$-test/Mann-Whitney test $\left(^{*}\right)$.

${ }^{b}$ Data are reported by $n$ (percentage of total); the chi-square test was used.

Statistically significant values in bold.

All hypothesis tests were 2-tailed, with $P<0.05$ denoting statistical significance. The Kolmogorov-Smirnov-test with a significance level of $5 \%$ was used to test continuous variables for the normality assumption. The chi-square test or Fisher exact-test was used to determine the differences of categorical variables between groups. Comparison between the variables was performed by paired-samples $t$-test/Wilcoxon at the beginning and end of the study in each group. To detect differences in continuous variables between the two groups, independentsamples $t$-test/Mann-Whitney was used. The main effects and interaction effects of the interventions were compared between groups using analysis of covariance (ANCOVA) with baseline measures as a covariate. The data were analyzed according to the intention-to-treat principle. Patients missing the final data were imputed. A multiple imputation procedure was used based on multiple imputations by chained equation. In the multiple imputation procedure, five imputed data sets were generated. The results of the five imputed data sets were pooled to obtain data estimates.

Collected information by food diaries was analyzed in the Nutritionist 4 software modified for Iranian foods (Karen Pharma \& Food Supplement Co., Tehran, Iran). Data from the food-record questionnaires were entered and analyzed by an expert dietitian.

\section{Ethics and Approvals}

The study protocol was approved by Shahid Beheshti Ethics Committee (IR.SBMU.NNFTRI.REC.1398.083), and it was registered at the Iranian Registry of Clinical Trials, with the registration number IRCT20100524004010N28.

\section{RESULTS}

\section{Patient Characteristics}

Between June 2020 and December 2020, 70 patients were recruited from two gastroenterology clinics and screened for this trial. From this initial group, 20 patients were excluded and the other 50 were enrolled and underwent the randomization process. Among the 50 included patients, 25 were assigned to the glutamine group and 25 were assigned to the placebo group. One patient was excluded from the glutamine group after discontinuing the study protocol, and two were lost to followup issues. Meanwhile, three patients from the control group were excluded due to non-compliance with the recommended diet. Therefore, 22 patients in the glutamine group and 22 patients in the control group completed the study protocol (Figure 1).

Table 1 shows the baseline characteristics of participants. The groups were similar in all characteristics except abdominal pain frequency $(p=0.022)$.

\section{Nutritional Data}

There was no significant difference in the nutritional compositions of the diet between the two groups (Table 2). All participants' dietary recalls demonstrated acceptable adherence to the diet. 
TABLE 2 | The mean daily nutrition information during the study in intervention groups.

\begin{tabular}{|c|c|c|c|c|c|c|}
\hline Parameter & \multicolumn{2}{|c|}{ Glutamine group $(n=25)$} & \multicolumn{2}{|c|}{ Control group $(n=25)$} & $P^{1}$ & $P^{2}$ \\
\hline Protein (gr) & $73.53 \pm 18.86$ & $71.81 \pm 22.96$ & $81.30 \pm 17.99$ & $77.96 \pm 16.64$ & 0.170 & 0.315 \\
\hline Fat (gr) & $67.40 \pm 12.99$ & $67.14 \pm 14.15$ & $67.08 \pm 13.59$ & $65.45 \pm 12.29$ & 0.936 & 0.778 \\
\hline Carbohydrates (gr) & $224.61 \pm 39.10$ & $223.33 \pm 39.33$ & $242.26 \pm 35.53$ & $239.70 \pm 44.88$ & 0.139 & 0.205 \\
\hline Lactose (gr) & $0.822 \pm 1$ & $0.622 \pm 1.02$ & $0.479 \pm 0.69$ & $0.277 \pm 0.65$ & 0.285 & 0.222 \\
\hline Excess fructose (gr) & $0.150 \pm 0.13$ & $0.143 \pm 0.09$ & $0.272 \pm 0.26$ & $0.168 \pm 0.13$ & 0.051 & 0.953 \\
\hline Polyols (gr) & $0.239 \pm 0.33$ & $0.191 \pm 0.34$ & $0.327 \pm 0.35$ & $0.254 \pm 0.41$ & 0.245 & 0.662 \\
\hline GOS (gr) & $0.125 \pm 0.09$ & $0.150 \pm 0.08$ & $0.131 \pm 0.05$ & $0.161 \pm 0.08$ & 0.798 & 0.657 \\
\hline FOS (gr) & $0.131 \pm 0.08$ & $0.186 \pm 0.10$ & $0.138 \pm 0.06$ & $0.193 \pm 0.08$ & 0.743 & 0.813 \\
\hline Total fructans (g) & $0.475 \pm 0.32$ & $0.599 \pm 0.28$ & $0.507 \pm 0.18$ & $0.644 \pm 0.30$ & 0.418 & 0.613 \\
\hline
\end{tabular}

Data are reported as mean $\pm S D$ and compared by sample t-test.

$P^{1}$ : between group at week 2 .

$P^{2}$ : between group at week 6 .

GOS, galaco-oligosaccharide; FOS, fructo-ologosaccharides; FODMAP, fermentable oligo- di- mono- saccharides and polyols. Statistically significant values are bolded.

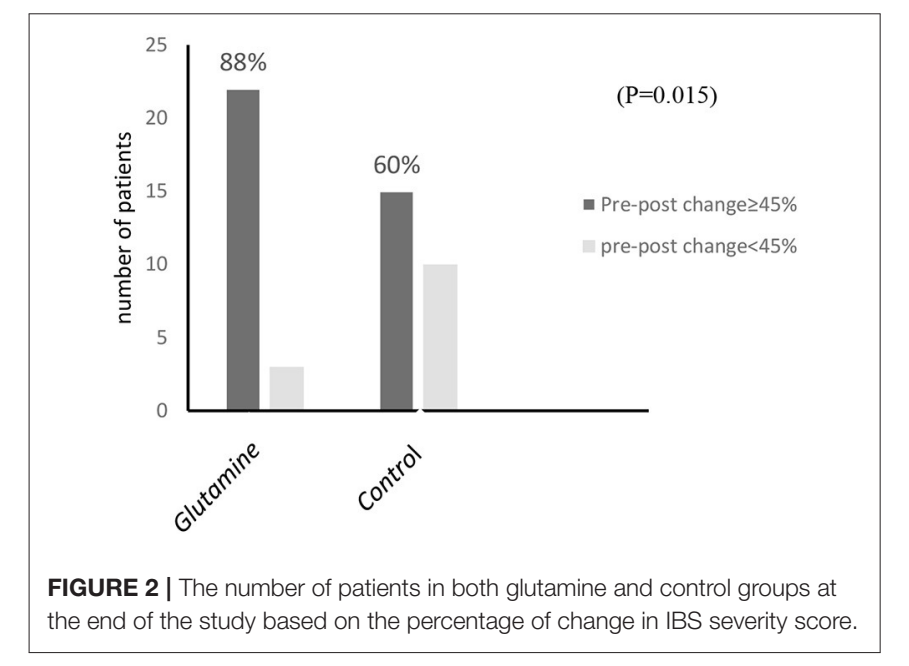

\section{Symptoms}

Improvement in IBS-severity score of more than $45 \%$ was observed in 22 of 25 participants $(88 \%)$ in the glutamine group, while it was only 15 of 25 participants $(60 \%)$ in the control group ( $P=0.015$; Figure 2$)$. The total scores of IBS-SS and the scores for individual items (abdominal pain intensity, abdominal pain frequency, abdominal distension, dissatisfaction with bowel habits, and interference with life), stool frequency, and consistency are shown in Table 3. Significant improvements were observed in these variables in both groups at the end of the study compared with the baseline $(p<0.001$ for total scores and individual items of IBS-SSS and $P=0.002, P=0.003$, respectively, for stool frequency and consistency).

Table 4 shows the comparison of pre-post-treatment changes between the glutamine and placebo groups. The glutamine group had significant changes in total IBS-severity score, dissatisfaction of bowel habit and interference with community function (58\% reduction; $P<0.001,57 \%$ reduction; $P<0.001,51 \%$ reduction; $P=0.043$, respectively). No adverse effect was reported in any group of interventions.

\section{Quality of Life}

Baseline quality of life scores was not significantly different between the two groups (Table 1). The QOL scores increased in both intervention groups $(+13 \pm 8.12, P<0.001$ in the glutamine group and $+11 \pm 5.12, P<0.001$ in the control group) at the end of the study compared with the baseline (Table 3 ). No significant difference was observed between groups at the end of the study $(P=0.353)$.

\section{DISCUSSION}

The present study demonstrated the superiority of adding a glutamine supplement to a low FODMAP diet in amelioration of IBS symptoms while also confirming the beneficial effects of low FODMAPs diets in IBS management (9).

It is well-known that a low FODMAPs diet ameliorates IBS symptoms by reducing luminal distension owing to the osmotic effects of FODMAPs and their rapid fermentation preferentially to hydrogen $(1,10,27)$. However, several studies have reported that increased intestinal permeability is another mechanism underlying IBS symptoms $(7,28)$. Moreover, it has been shown that this disturbance in gut integrity is related to decreased glutamine synthetase levels in patients with IBS (13). This, in turn, results in visceral hypersensitivity, leading to increased gastrointestinal symptoms $(7,13)$. Experimental evidence has also shown that glutamine improves intestinal permeability $(29,30)$. 
TABLE 3 | Gastrointestinal symptoms and quality of life in patients with irritable bowel syndrome.

\begin{tabular}{|c|c|c|c|c|c|c|}
\hline Parameter & \multicolumn{3}{|c|}{ Glutamine group $(n=25)$} & \multicolumn{3}{|c|}{ Control group $(n=25)$} \\
\hline Abdominal pain intensity & $65.91 \pm 25.80$ & $29.09 \pm 14.36$ & $<0.001$ & $56.36 \pm 15.44$ & $29.55 \pm 11.64$ & $<0.001$ \\
\hline Abdominal pain frequency & $52.73 \pm 29.14$ & $20 \pm 10.69$ & $<0.001$ & $35 \pm 18.71$ & $18.64 \pm 8.33$ & $<0.001$ \\
\hline Abdominal distension & $56.14 \pm 22.30$ & $19.55 \pm 8.58$ & $<0.001$ & $66.82 \pm 12.20$ & $26.14 \pm 9.50$ & $<0.001$ \\
\hline Interference with life & $69.32 \pm 22.43$ & $33.64 \pm 13.64$ & $<0.001$ & $70.91 \pm 10.19$ & $41.59 \pm 9.31$ & $<0.001$ \\
\hline Stool frequency (no./day) & $2.95 \pm 2.19$ & $1.59 \pm 0.796$ & 0.002 & $2.68 \pm 2.06$ & $1.68 \pm 0.894$ & 0.002 \\
\hline Stool consistency & $5.14 \pm 1.70$ & $4.00 \pm 0.617$ & 0.003 & $4.55 \pm 1.62$ & $3.82 \pm 0.907$ & 0.003 \\
\hline Quality of life & $63.23 \pm 19.99$ & $76.32 \pm 16.74$ & $<0.001$ & $65.41 \pm 10.44$ & $76.45 \pm 9.86$ & $<0.001$ \\
\hline
\end{tabular}

Values are reported as mean $\pm S D$.

$P^{1}$ : Obtained from the Wilcoxon test for comparison of data between the beginning and end of the study.

$P^{2}$ : Obtained from the paired samples t-test for comparison of data between the beginning and end of the study.

TABLE 4 | Effects of intervention on studied outcomes.

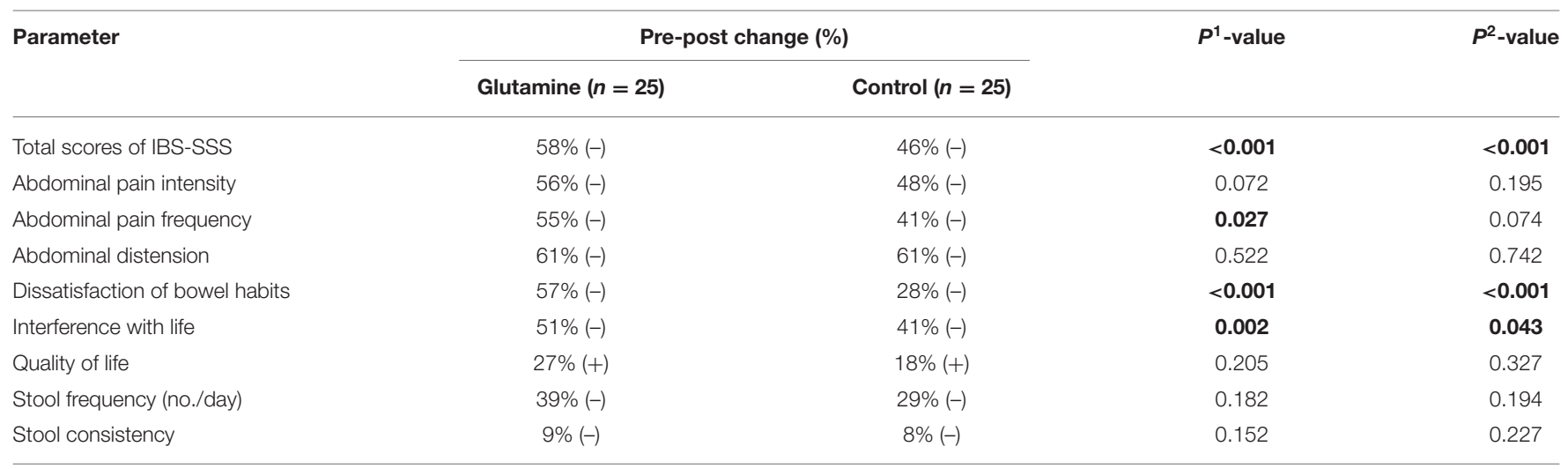

$P^{1}$-value: Obtained from two-sample t-test.

$P^{2}$-value: Obtained from ANCOVA test adjusted for the baseline values between studied groups. Statistically significant values are bolded.

On the other hand, low-grade inflammation has been observed in the intestinal mucosa of patients with IBS, especially those with increased intestinal permeability (31). Evidence shows that glutamine has anti-inflammatory properties, as it inhibits the activation of nuclear factor $\kappa \mathrm{B}(\mathrm{NF}-\kappa \mathrm{B})$, signal transducer and activator of transcription (STAT), and inflammatory cytokines such as IL-6, TNF- $\alpha$, and IL-8 (12).

Zhou et al. (32) reported that the up-regulation of microRNA29 in the colonic mucosa of patients with IBS-D reduces claudin-1 levels, which leads to increased intestinal permeability. Claudin-1 is an integral component of the structure of tight junctions and plays a crucial role in regulating epithelial barrier function (33). Therefore, the alteration of tight junction proteins might initiate IBS and contribute to visceral hypersensitivity $(34,35)$. Meanwhile, in an ex-vivo study evaluating the effects of glutamine on claudin- 1 tight junction proteins, colonic biopsies from patients with IBS-D were incubated in cell cultures with glutamine, and the results indicated increased claudin-1 expression (14).

Studies investigating the effects of glutamine supplementation on the severity of gastrointestinal symptoms in patients with IBS are scarce. In line with our study, Zhou et al. (21) found that oral glutamine supplementation normalized intestinal permeability and improved gastrointestinal symptoms in post-infectious IBS. In this study, the patients did not receive dietary advice, so the changes in IBS symptoms were less than our study.

Moreover, recent studies have reported the potential role of intestinal microbiota in the pathophysiology of IBS (3638). For instance, gut microbiota can affect motor function, hypersensitivity, and immune activity in the gut (resulting in low-grade inflammation), leading to the development of IBS or the exacerbation of gastrointestinal symptoms (37, 39). A recent review of clinical, in vitro, and in vivo studies reported that glutamine affects gut microbiota community and composition through several mechanisms. Therefore, it can be used to manage some conditions such as bacterial translocation, inflammation, and constipation (18). Furthermore, Zhang et al. reported that glutamine supplementation in constipated animals improves intestinal function and ameliorates constipation by modulating gut microbiota through increasing intestinal friendly microbiota levels (19). 
The protective and therapeutic roles of glutamine have also been reported in clinical trials for other gastrointestinal diseases (40-42). Glutamine could possibly improve the condition of patients with IBS by regulating intestinal permeability via increased tight junction proteins expression, modulating the inflammatory response, oxidative stress, or innate immune response, and also altering intestinal microbiota (29).

This study has some advantages; According to our research, this is the first clinical trial that assessed the superiority of adding glutamine supplement to low FODMAPs in the management of patients with IBS. Preparing individually low FODMAPs diet, assessment of dietary composition, FODMAPs content, and also assessment of dietary adherence during the intervention were other strengths of this study. A limitation of this study was that patients were not followed up after the study ended. Given that national local factors affect the composition of FODMAPs in foods (27), measuring the content of FODMAPs using nonlocalized data was another limitation of our study.

\section{CONCLUSION}

In conclusion, this randomized, double-blind, placebocontrolled trial has shown the superiority of adding glutamine supplementation to a low FODMAPs diet in amelioration of IBS symptoms, while confirming the beneficial effects of a low FODMAPs diet in IBS management. Further studies are needed to find the optimum dosage of glutamine supplementation for IBS management.

\section{REFERENCES}

1. Portincasa P, Bonfrate L, de Bari O, Lembo A, Ballou S. Irritable bowel syndrome and diet. Gastroenterol Rep. (2017) 5:11-9. doi: 10.1093/gastro/gow047

2. Wilson B, Rossi M, Dimidi E, Whelan K. Prebiotics in irritable bowel syndrome and other functional bowel disorders in adults: a systematic review and meta-analysis of randomized controlled trials. Am J Clin Nutr. (2019) 109:1098-111. doi: 10.1093/ajcn/nqy376

3. Defrees DN, Bailey J. Irritable bowel syndrome: epidemiology, pathophysiology, diagnosis, and treatment. Prim Care. (2017) 44:655. doi: 10.1016/j.pop.2017.07.009

4. Ford AC, Moayyedi P, Chey WD, Harris LA, Lacy BE, Saito YA, et al. American College of Gastroenterology monograph on management of irritable bowel syndrome. Am J Gastroenterol. (2018) 113:1-18. doi: 10.1038/s41395-018-0084-x

5. Peery AF, Dellon ES, Lund J, Crockett SD, McGowan CE, Bulsiewicz WJ, et al. Burden of gastrointestinal disease in the United States: 2012 update. Gastroenterology. (2012) 143:1179-87.e3. doi: 10.1053/j.gastro.2012.08.002

6. Gwee KA, Ghoshal UC, Chen M. Irritable bowel syndrome in Asia: pathogenesis, natural history, epidemiology, and management. J Gastroenterol Hepatol. (2018) 33:99-110. doi: 10.1111/jgh.13987

7. Zhou Q, Zhang B, Verne GN. Intestinal membrane permeability and hypersensitivity in the irritable bowel syndrome. Pain. (2009) 146:416. doi: 10.1016/j.pain.2009.06.017

8. Altobelli E, Del Negro V, Angeletti PM, Latella G. Low-FODMAP diet improves irritable bowel syndrome symptoms: a meta-analysis. Nutrients. (2017) 9:940. doi: 10.3390/nu9090940

9. Halmos EP, Power VA, Shepherd SJ, Gibson PR, Muir JG. A diet low in FODMAPs reduces symptoms of irritable bowel syndrome. Gastroenterology. (2014) 146:67-75.e5. doi: 10.1053/j.gastro.2013.09.046

\section{DATA AVAILABILITY STATEMENT}

The raw data supporting the conclusions of this article will be made available by the authors, without undue reservation.

\section{ETHICS STATEMENT}

The studies involving human participants were reviewed and approved by NNFTRI. The patients/participants provided their written informed consent to participate in this study.

\section{AUTHOR CONTRIBUTIONS}

SR and AH: conceptualized and designed the study and wrote the manuscript. SR and BR: analyzed the data. SR, NE-D, SA, FM, MA, MT, and SA: collected the data. EH and $\mathrm{AH}$ : interpreted the data and provided professional comments. AH: critically revised the manuscript for intellectual content, data accuracy, and had responsibility for the final content. All authors have read and approved the final manuscript.

\section{FUNDING}

This study was financially supported by Shahid Beheshti University of Medical Sciences, Tehran, Iran. The funder did not play any role in study design and interference.

10. Whelan K, Martin LD, Staudacher HM, Lomer MC. The low FODMAP diet in the management of irritable bowel syndrome: an evidence-based review of FODMAP restriction, reintroduction and personalisation in clinical practice. J Hum Nutr Diet. (2018) 31:239-55. doi: 10.1111/jhn.12530

11. Abhari K, Saadati S, Hosseini-Oskouiee F, Yari Z, Hosseini H, Sohrab G, et al. Is Bacillus coagulans supplementation plus low FODMAP diet superior to low FODMAP diet in irritable bowel syndrome management? Eur J Nutr. (2020) 59:2111-7. doi: 10.1007/s00394-019-02060-y

12. Kim M-H, Kim H. The roles of glutamine in the intestine and its implication in intestinal diseases. Int J Mol Sci. (2017) 18:1051. doi: 10.3390/ijms18051051

13. Zhou Q, Souba WW, Croce CM, Verne GN. MicroRNA-29a regulates intestinal membrane permeability in patients with irritable bowel syndrome. Gut. (2010) 59:775-84. doi: 10.1136/gut.2009.181834

14. Bertrand J, Ghouzali I, Guérin C, Bôle-Feysot C, Gouteux M, Déchelotte P, et al. Glutamine restores tight junction protein claudin-1 expression in colonic mucosa of patients with diarrhea-predominant irritable bowel syndrome. $J$ Parent Enteral Nutr. (2016) 40:1170-6. doi: 10.1177/0148607115587330

15. Wang W, Qiao S, Li D. Amino acids and gut function. Amino Acids. (2009) 37:105-10. doi: 10.1007/s00726-008-0152-4

16. Dai Z-L, Li X-L, Xi P-B, Zhang J, Wu G, Zhu W-Y. L-Glutamine regulates amino acid utilization by intestinal bacteria. Amino Acids. (2013) 45:50112. doi: $10.1007 / \mathrm{s} 00726-012-1264-4$

17. Dai Z-L, Wu G, Zhu W-Y. Amino acid metabolism in intestinal bacteria: links between gut ecology and host health. Front Biosci. (2011) 16:176886. doi: $10.2741 / 3820$

18. Perna S, Alalwan TA, Alaali Z, Alnashaba T, Gasparri C, Infantino $\mathrm{V}$, et al. The role of glutamine in the complex interaction between gut microbiota and health: a narrative review. Int J Mol Sci. (2019) 20:5232. doi: 10.3390/ijms20205232

19. Zhang $\mathrm{Y}, \mathrm{Lu} \mathrm{T}$, Han L, Zhao L, Niu Y, Chen H. L-glutamine supplementation alleviates constipation during late gestation of mini 
sows by modifying the microbiota composition in feces. BioMed Res Int. (2017). 2017:2861. doi: 10.1155/2017/4862861

20. Drossman DA. Functional gastrointestinal disorders: history, pathophysiology, clinical features, and Rome IV. Gastroenterology. (2016) 150:1262-79.e2. doi: 10.1053/j.gastro.2016.02.032

21. Zhou Q, Verne ML, Fields JZ, Lefante JJ, Basra S, Salameh H, et al. Randomised placebo-controlled trial of dietary glutamine supplements for postinfectious irritable bowel syndrome. Gut. (2019) 68:996-1002. doi: 10.1136/gutjnl-2017-315136

22. Biesiekierski JR, Rosella O, Rose R, Liels K, Barrett J, Shepherd SJ, et al. Quantification of fructans, galacto-oligosacharides and other short-chain carbohydrates in processed grains and cereals. J Hum Nutr Diet. (2011) 24:154-76. doi: 10.1111/j.1365-277X.2010.01139.x

23. Muir JG, Rose R, Rosella O, Liels K, Barrett JS, Shepherd SJ, et al. Measurement of short-chain carbohydrates in common Australian vegetables and fruits by high-performance liquid chromatography (HPLC). J Agric Food Chem. (2009) 57:554-65. doi: 10.1021/jf802700e

24. Lembo A, Ameen VZ, Drossman DA. Irritable bowel syndrome: toward an understanding of severity. Clin Gastroenterol Hepatol. (2005) 3:71725. doi: 10.1016/S1542-3565(05)00157-6

25. Gholamrezaei A, Zolfaghari B, Farajzadegan Z, Nemati K, Daghaghzadeh $\mathrm{H}$, Tavakkoli $\mathrm{H}$, et al. Linguistic validation of the irritable bowel syndrome-quality of life questionnaire for Iranian patients. Acta Med Iran. (2011) 49:390-5.

26. Blake M, Raker J, Whelan K. Validity and reliability of the Bristol Stool Form Scale in healthy adults and patients with diarrhoeapredominant irritable bowel syndrome. Aliment Pharmacol Ther. (2016) 44:693-703. doi: 10.1111/apt.13746

27. Varney J, Barrett J, Scarlata K, Catsos P, Gibson PR, Muir JG. FODMAPs: food composition, defining cutoff values and international application. $J$ Gastroenterol Hepatol. (2017) 32:53-61. doi: 10.1111/jgh.13698

28. Dunlop SP, Hebden J, Campbell E, Naesdal J, Olbe L, Perkins AC, et al. Abnormal intestinal permeability in subgroups of diarrheapredominant irritable bowel syndromes. Am J Gastroenterol. (2006) 101:1288-94. doi: 10.1111/j.1572-0241.2006.00672.x

29. Achamrah N, Déchelotte P, Coëffier M. Glutamine and the regulation of intestinal permeability: from bench to bedside. Curr Opin Clin Nutr Metabolic Care. (2017) 20:86-91. doi: 10.1097/MCO.0000000000000339

30. dos Santos RdGC, Viana ML, Generoso SV, Arantes RE, Davisson Correia MIT, Cardoso VN. Glutamine supplementation decreases intestinal permeability and preserves gut mucosa integrity in an experimental mouse model. J Parent Enteral Nutr. (2010) 34:408-13. doi: 10.1177/0148607110362530

31. Ng QX, Soh AYS, Loke W, Lim DY, Yeo WS. The role of inflammation in irritable bowel syndrome (IBS). J Inflamm Res. (2018) 11:3459. doi: $10.2147 /$ JIR.S174982

32. Zhou Q, Costinean S, Croce CM, Brasier AR, Merwat S, Larson SA, et al. MicroRNA 29 targets nuclear factor- $\mathrm{B}$-repressing factor and Claudin 1 to increase intestinal permeability. Gastroenterology. (2015) 148:15869.e8. doi: 10.1053/j.gastro.2014.09.037

33. Pope JL, Bhat AA, Sharma A, Ahmad R, Krishnan M, Washington MK, et al. Claudin-1 regulates intestinal epithelial homeostasis through the modulation of Notch-signalling. Gut. (2014) 63:622-34. doi: 10.1136/gutjnl-2012-304241
34. Bertiaux-Vandaële N, Youmba SB, Belmonte L, Lecleire S, Antonietti M, Gourcerol G, et al. The expression and the cellular distribution of the tight junction proteins are altered in irritable bowel syndrome patients with differences according to the disease subtype. Am J Gastroenterol. (2011) 106:2165-73. doi: 10.1038/ajg.20 11.257

35. Martínez C, Vicario M, Ramos L, Lobo B, Mosquera JL, Alonso $\mathrm{C}$, et al. The jejunum of diarrhea-predominant irritable bowel syndrome shows molecular alterations in the tight junction signaling pathway that are associated with mucosal pathobiology and clinical manifestations. Am J Gastroenterol. (2012) 107:736-46. doi: 10.1038/ajg.20 11.472

36. Collins SM. A role for the gut microbiota in IBS. Nat Rev Gastroenterol Hepatol. (2014) 11:497-505. doi: 10.1038/nrgastro.2014.40

37. Dupont $\mathrm{H}$. evidence for the role of gut microbiota in irritable bowel syndrome and its potential influence on therapeutic targets. Aliment Pharmacol Ther. (2014) 39:1033-42. doi: 10.1111/apt.12728

38. Lee $\mathrm{KN}$, Lee OY. Intestinal microbiota in pathophysiology and management of irritable bowel syndrome. World J Gastroenterol. (2014) 20:8886. doi: 10.3748/wjg.v20.i10.2456

39. Jeffery IB, Quigley EM, Öhman L, Simrén M, O’Toole PW. The microbiota link to irritable bowel syndrome: an emerging story. Gut Microbes. (2012) 3:572-6. doi: 10.4161/gmic. 21772

40. Benjamin J, Makharia G, Ahuja V, Rajan KA, Kalaivani M, Gupta SD, et al. Glutamine and whey protein improve intestinal permeability and morphology in patients with Crohn's disease: a randomized controlled trial. Dig Dis Sci. (2012) 57:1000-12. doi: 10.1007/s10620-011-1947-9

41. Schanuel CM, Dias EN, Ferreira AP. Glutamine as a therapeutic strategy in inflammatory bowel diseases: a systematic review. Gastroint Hepatol Dig Dis. (2019) 2:1-6. doi: 10.33425/2639-9334.1033

42. Xue P, Deng L-H, Xia Q, Zhang Z-D, Hu W-M, Yang X-N, et al. Impact of alanyl-glutamine dipeptide on severe acute pancreatitis in early stage. World $J$ Gastroenterol. (2008) 14:474. doi: 10.3748/wjg.14.474

Conflict of Interest: The authors declare that the research was conducted in the absence of any commercial or financial relationships that could be construed as a potential conflict of interest.

Publisher's Note: All claims expressed in this article are solely those of the authors and do not necessarily represent those of their affiliated organizations, or those of the publisher, the editors and the reviewers. Any product that may be evaluated in this article, or claim that may be made by its manufacturer, is not guaranteed or endorsed by the publisher.

Copyright (C) 2021 Rastgoo, Ebrahimi-Daryani, Agah, Karimi, Taher, Rashidkhani, Hejazi, Mohseni, Ahmadzadeh, Sadeghi and Hekmatdoost. This is an open-access article distributed under the terms of the Creative Commons Attribution License (CC $B Y)$. The use, distribution or reproduction in other forums is permitted, provided the original author(s) and the copyright owner(s) are credited and that the original publication in this journal is cited, in accordance with accepted academic practice. No use, distribution or reproduction is permitted which does not comply with these terms. 